\title{
A Wavelength Identification System Using an Optical Source Spectrum
}

\author{
Jaemyoung Lee
}

\begin{abstract}
This paper proposes an optical wavelength identification system without any wavelength analyzing instrument which is vulnerable to environment vibration and impact. The proposed system obtains wavelength information through the conversion from measured intensity on a photodetector to an optical wavelength using spectrum characteristics of an optical source in the system. Data processing unit of the proposed system has data of intensity vs, wavelength of the optical source. The proposed system is advantageous in terms of overall cost, system volume, and robustness to mechanical impact.
\end{abstract}

Keywords - Optical filter, Optical intensity, Optical spectrum, Wavelength.

\section{INTRODUCTION}

Optical filters are key components in lots of applications such as optical sensors, optical communications, optical measurement systems, microwave photonic systems, etc. Recently optical technologies have developed to be able to implement optical filters for low pass filters, high pass filters, band pass filters, and band rejection filters [1]-[4]. For an optical sensor system which makes use of optical wavelength variation as an indication in the change of some physical quantity such as temperature, pressure, strain, chemical component variation, and so on [1]-[5]. For those purposes, Fiber Bragg Grating (FBG) filter as an optical sensor have mostly been used [6]-[8]. The FBG filter has evolved into many advanced kinds of FBG devices for various application areas. To use the FBG filter in a sensor system, the sensor system requires certain optical wavelength measurement instrument to monitor the optical wavelength variation [3]. The optical wavelength monitoring system can be implemented with a tunable filter, or diffraction grating, of which precision and stability is affected by misalignment, external vibration, or mechanical impact. To overcome those problems, the proposed system uses photodetectors which is not only robust to mechanical impact but not requiring accurate alignment, to identify a wavelength of a beam from a fiber sensor.

In this paper, we propose a new wavelength measurement system which does not require optical wavelength measurement devices vulnerable to environment vibration and impact, such as tunable filters and diffraction gratings. The proposed system obtains wavelength information through the

Manuscript received Mar. 16, 2017.

Jaemyoung Lee is with the Department of Electronic Engineering, Korea Polytechnic University, 2121 Jungwang Shihung Kyunggi, 15073 Korea(ROK) (e-mail: lee@ kpu.ac.kr). conversion from measured intensity on photodetector to optical wavelength using the spectrum characteristics of the optical source in the system.

Principles of the proposed wavelength identification system

An optical sensor system is generally composed of an optical source, an optical filter and a detection device. For a system which uses the wavelength variation as an indication of change in physical quantities such as temperature, pressure, or strain in the observed materials, the system requires a wavelength measurement instrument which requires high precision alignment.

The proposed system uses the optical wavelength characteristic of the optical source in the sensing system to eliminate the fine alignment of the wavelength measurement instrument. Let's assume that the wavelength spectrum of the optical source is like that of Fig. 1, where the optical wavelength spectrum is assumed as a Gaussian shape. Note that the optical intensity in the optical spectrum of the source is different for different wavelength, even though two wavelengths correspond to the same intensity. That is, , if we use half of the wavelength region of the source spectrum, we could identify the wavelength by measuring optical intensity incident on the photodetector in the proposed system which employs a photodetector in receiving unit rather than an optical wavelength analyzing instrument. The intensity information is sampled for different wavelength as shown in Fig. 2 and stored in the data processing unit. When optical intensity incident on the photodetector is the

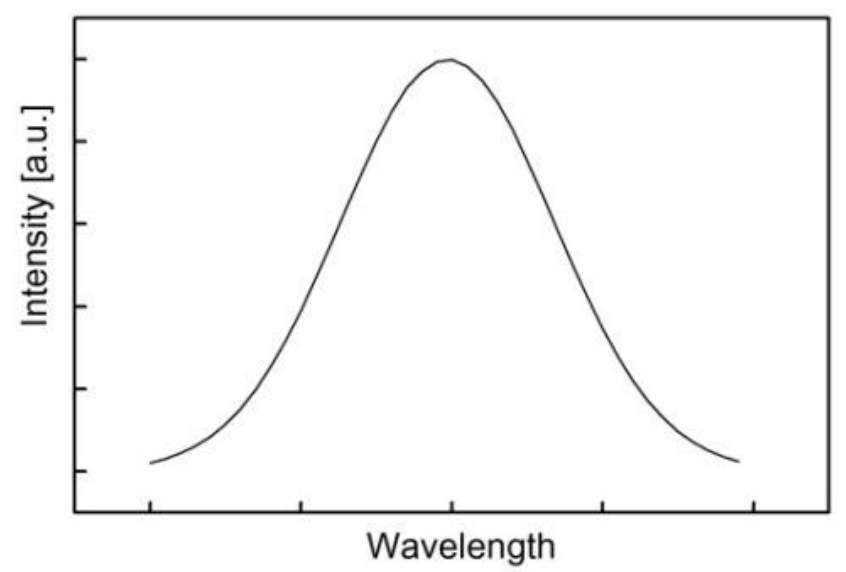

Fig. 1. The assumed wavelength spectrum of optical source in the proposed system. 


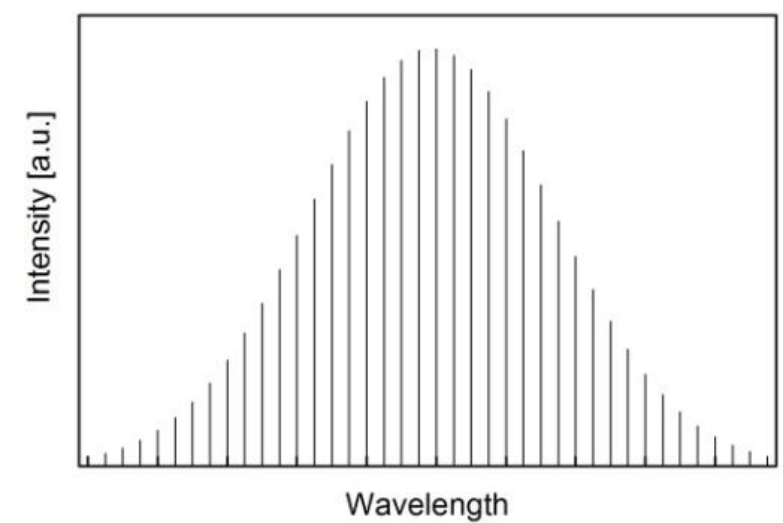

Fig. 2. The sampled wavelength spectrum for the optical source in the system.

value between two intensities in the optical intensity vs. wavelength table, the wavelength corresponding to the incident intensity can be calculated through interpolation calculation. In the case of Fig. 3, the wavelength $\lambda x$ corresponding to the intensity Px can be calculated as follows,

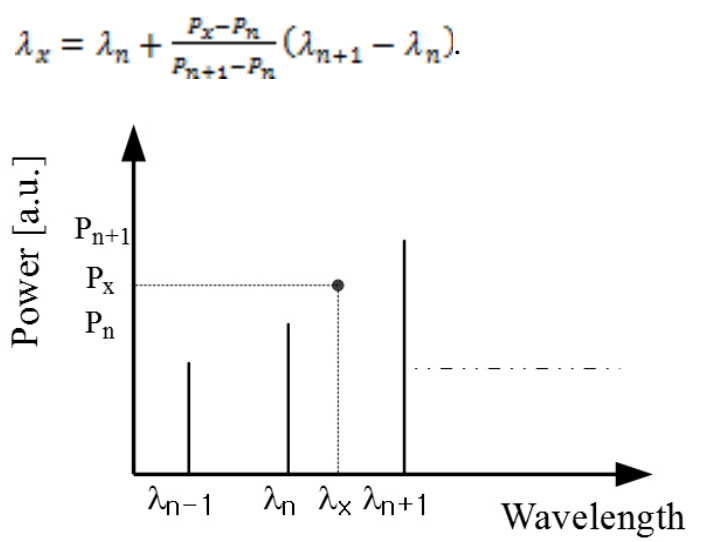

Fig. 3. The sampled wavelength spectrum for the optical source in the system.

\section{PROPOSED SYSTEM TO IDENTIFY THE WAVELENGTH USING INTENSITY OF THE OPTICAL SOURCE}

The proposed system in which the intensity on a photodetector can identify the corresponding wavelength is shown in Fig. 4. In the proposed system, the wavelength

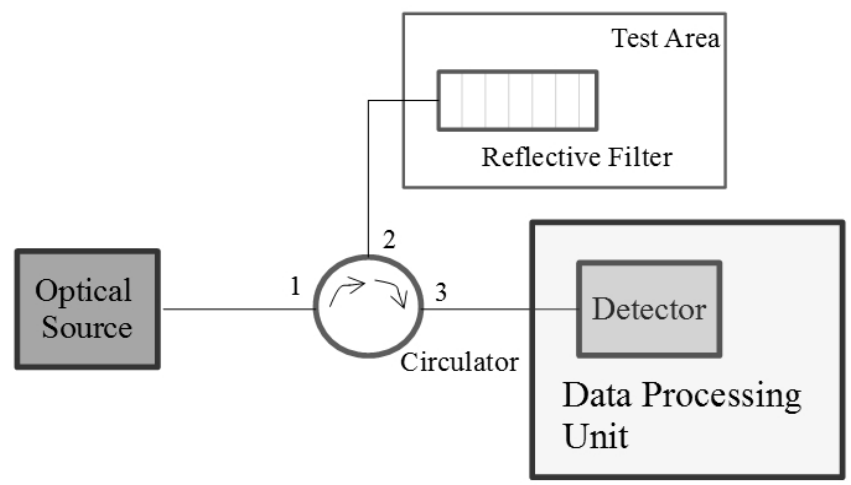

Fig. 4. The proposed optical system to identify the wavelength through the intensity incident on a photodetector

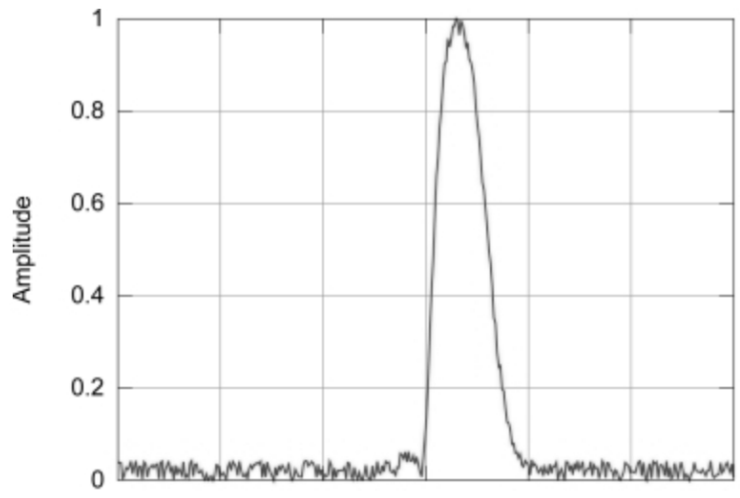

Wavelength [nm]

Fig. 5. The sampled wavelength spectrum for the optical source in the system.

spectrum of the optical source is measured and stored in the data processing unit. At the sensing area, a fiber sensor which is a reflective filter in the proposed system is installed. Fig. 5 shows the typical optical spectrum of a reflective filter for a fiber sensor. In Fig. 4, the reflected wavelength by the reflective filter propagates into the port 2 of the circulator and travels to the detector through the port 3 . Some change in temperature, pressure or strain around the test area reflects different wavelength coming from the optical source. The reflected wavelength which is dependent on the current physical quantity, such as temperature, pressure, or strain, in the test area can be different from the previous wavelength if there is any change in physical quantity of the test area. Because intensity is different for different wavelength, the data processing unit receives the different intensity through the detector compared with the previous intensity, if there is any wavelength change in incident beam on the detector, and identifies the wavelength based on the intensity vs. wavelength table in the data processing unit. The proposed system only use half side, left or right side, on the wavelength spectrum, in Fig. 2 which shows the stored optical source spectrum in the data processing unit, because there are two wavelengths for an intensity value.

Fig. 6 shows the modified proposed system to use the whole wavelength range of the optical source in the proposed system. By employing the reflective filter2 of which reflection band is a half side, left or right side, on the wavelength spectrum, and of which reflectivity is very small amount of incident beam, such

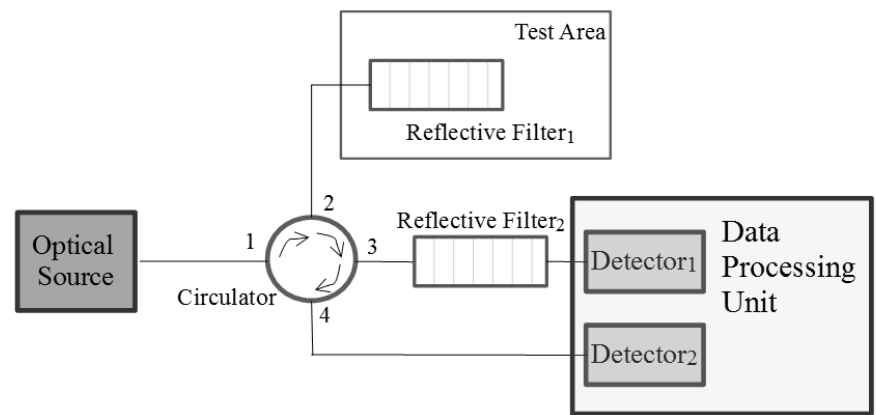

Fig. 6. The proposed optical system to identify the wavelength through the intensity incident on a photodetector which can use the whole wavelength range of the optical source 
as $1 \%$, the detector 2 in Fig. 6 can discriminate whether the incident beam on the detector 1 comes from the left side, or the right side of the wavelength spectrum

\section{CONCLUSION}

A wavelength identification system is proposed. The proposed system can identify wavelength of the incoming beam on the detector using intensity information of the optical source in the system without any wavelength analyzing instrument. The proposed system is advantageous in terms of overall cost, system volume, implementation, and robustness to mechanical impact.

\section{REFERENCES}

[1] A. Emadi, H.Wu, S. Grabarnik, G.De Graaf, K. Hedsten, P. Enoksson, J. H. Correia, R. F. Wolffenbuttel, "Fabrication and characterization of IC-Compatible Linear Variable Optical Filters with application in a micro-spectrometer," Sensors and Actuators, vol. 162, pp. 400-405, 2010
[2] A. Emadi, S. Grabarnik, H.Wu, G.de Graaf, R. F. Wolffenbuttel, "Spectral measurement with a linear variable filter using a LMS algorithm," Procedia Engineering, vol. 5, pp. 504-507, 2010.

[3] S. Weidong, L. Xiangdong, H. Biqin, Z. Yong, L. Xu, G. Peifu, “Analysis on the tunable optical properties of MOEMS filter based on Fabry-Perot Cavity," Optics Communications, vol. 239, pp. 153-160, $2004 .$.

[4] G. Lammel, S. Schweizer, P. Renaud, "Microspectrometer based on a tunable optical filter of porous silicon," Sensors and Actators A, vol. 92, pp. 52-59, 2001..

[5] M. S. Islam, A. B. Mohammad, "The effect of elastic fatigue on the output transmission of a Mach-Zehnder interferometer comb filter," Optical Fiber Technology, vol. 20, pp. 299-302, 2014.

[6] Y. Miao, B. Liu, K. Zhang, Q. Zhao, "Linear edge and temperature characteristic of tilted fiber Bragg gratings cladding-mode envelope," Optical Fiber Technology, vol. 17, pp. 286-290, 2011.

[7] J. H. Osório, R. Oliveira, S. Aristilde, G. Chesini, M. A. R. Franco, R. N. Nogueira, C. M. B. Cordeiro, "Bragg gratings in surface-core fibers: Refractive index and directional curvature sensing," Optical Fiber Technology, vol. 34, pp. 86-90, 2017.

[8] M.-Y. Chen, J. Wei, Y. Sheng, N.-F. Ren, "Design and optimization of fundamental mode filters based on long-period fiber gratings," Optical Fiber Technology, vol. 30, pp. 89-94, 2016. 